Table 1. Comparative analysis using chi-square or Fischer test

\begin{tabular}{lccc}
\hline & SARD & Non-SARD & p-value \\
\hline Arthralgia, $\mathrm{n}$ & 10 & 43 & 0.001 \\
Arthritis, $\mathrm{n}$ & 14 & 0 & $<0.001$ \\
Raynaud Phenomenon, $\mathrm{n}$ & 7 & 5 & 0.114 \\
Raised Inflammation parameters, $\mathrm{n}$ & 6 & 3 & 0.045 \\
Anaemia, $\mathrm{n}$ & 1 & 2 & 1.000 \\
Leukopenia, $\mathrm{n}$ & 3 & 1 & 0.131 \\
\hline
\end{tabular}

Conclusion: We concluded that $64.2 \%$ of patients with positive DFS70 did not present a SARD and if we only consider patients with isolated anti-DFS70, $77.1 \%$ didn't present a SARD. Therefore, in our study, $22.9 \%$ of the patients presented a SARD, which was associated with some clinical features like arthritis or raised inflammatory parameters $(p<0.05)$. Although isolated anti-DFS70 are not specific of a particular condition, our study supports that it can be used as a negative predictor of SARD, if a correlation with clinical and laboratory features is made.

REFERENCES:

[1] Leuchten, N. et al. Performance of Antinuclear Antibodies for Classifying Systemic Lupus Erythematosus: A systematic literature review and meta-regression of diagnostic data. Arthritis Care Res. 70, 428-438 (2018).

[2] Carter JB, Carter S, Saschenbrecker S, Goeckeritz BE (2018) Recognition and relevance of anti-DFS70 autoantibodies in routine antinuclear autoantibodies testing at a community hospital.

Disclosure of Interests: None declared

DOI: 10.1136/annrheumdis-2021-eular.1201

\section{POS0264 THE EMERGING ROLE OF MAGNETIC RESONANCE IMAGING IN INTERSTITIAL LUNG DISEASE IN SYSTEMIC SCLEROSIS: EVIDENCE FOR ULTRA SHORT TE AND COMPRESSED SENSING VIBE ACQUISITIONS AS PROMISING TOOLS FOR THE EVALUATION OF PARENCHYMAL ALTERATIONS}

N. Landini' ${ }^{1}$, M. Orlandi ${ }^{2}$, M. Occhipinti ${ }^{3}$, C. Nardi ${ }^{3}$, L. Tofani ${ }^{2}$, S. BellandoRandone $^{2}$, C. Bruni ${ }^{2}$, M. Matucci-Cerinic ${ }^{2}$, G. Morana ${ }^{1}$, S. Colagrande ${ }^{3} .{ }^{1} \mathrm{Ca}^{\prime}$ Foncello General Hospital, Department of Radiology, Treviso, Italy; ${ }^{2}$ University of Florence, Department of Experimental and Clinical Medicine, Florence, Italy; ${ }^{3}$ University of Florence-Azienda Ospedaliero-Universitaria Careggi, Department of Experimental and Clinical Biomedical Sciences,

Florence, Italy

Background: Interstitial lung disease (ILD) is a frequent complication and the major cause of death in Systemic sclerosis (SSc). Computed tomography (CT) is the gold standard imaging technique to assess ILD but is burdened by exposure to ionizing radiations that limits its use for the follow-up. MRI sequences with Ultra Short Echo Time (UTE) are promising for ILD.

Objectives: We tested two MRI sequences, UTE Spiral VIBE and Compressing Sensing (CS) VIBE, in SSc-ILD, in comparison to chest CT.

Methods: SSc patients with suspected-ascertained ILD were evaluated for undergoing CT-MRI examinations in the same day. Two radiologists visually scored the extent of ground glass opacities (GGO), reticulations, honeycombing and consolidations on CT-MRI. The sum of alteration was assumed as ILD extent. A quantitative texture analysis (qCT) was also performed on CT. Cohen's $\mathrm{k}$ was adopted for interreader concordance in ILD detection. MRI sensitivity and specificity in ILD detection were evaluated. Lin's concordance was adopted to compare extent analysis between readers and between CT (visual and qCT analysis) and MRI sequences.

Results: 54 patients performed both CT and MRI. MRI interreader concordance was moderate in ILD detection, while ILD and GGO extent analysis showed good or very good concordance. UTE Spiral VIBE had a sensitivity and specificity in ILD detection of $95.8 \%$ and $77.8 \%$, while alterations extent analysis obtained a very good concordance with CT for ILD and GGO. CS VIBE showed a sensitivity and specificity in ILD detection of $46.7 \%$ and $95.0 \%$, but a slight or fair concordance with CT in all alterations' extent analysis.

Conclusion: MRI UTE Spiral VIBE sequences are helpful in the evaluation of SSc-ILD. Larger cohorts of patients will be needed to confirm that MRI may be useful in clinical practice, reducing the radiological load of chest CT

\section{REFERENCES:}

[1] Romei C, Turturici L, Tavanti L, et al. The use of chest magnetic resonance imaging in interstitial lung disease: a systematic review. Eur Respir Rev. 2018;27(150):180062. Doi:10.1183/16000617.0062-2018

[2] Miller GW, Mugler JP, Sá RC, Altes TA, Prisk GK, Hopkins SR. Advances in functional and structural imaging of the human lung using proton MRI. NMR Biomed. 2014;27(12):1542-1556. doi:10.1002/nbm.3156

[3] Pinal-Fernandez I, Pineda-Sanchez V, Pallisa-Nuñez E, et al. Fast $1.5 \mathrm{~T}$ chest MRI for the assessment of interstitial lung disease extent secondary to systemic sclerosis. Clin Rheumatol. 2016;35(9):2339-2345. doi:10.1007/ s10067-016-3267-0

[4] Ohno $\mathrm{Y}$, Koyama $\mathrm{H}$, Yoshikawa T, et al. Pulmonary high-resolution ultrashort TE MR imaging: Comparison with thin-section standard- and low-dose computed tomography for the assessment of pulmonary parenchyma diseases: Pulmonary MRI with UTE in Pulmonary Disease. J Magn Reson Imaging 2016;43(2):512-532. doi:10.1002/jmri.25008

Disclosure of Interests: None declared

DOI: 10.1136/annrheumdis-2021-eular.3253

\section{POS0265 CLINICAL AND ULTRASONOGRAPHIC ENTHESITIS IN INFLAMMATORY BOWEL DISEASE WITH AND WITHOUT PSORIASIS}

G. Citriniti ${ }^{1}$, A. Soriano ${ }^{2}$, E. Bertolini ${ }^{2}$, G. Sandri ${ }^{3}$, A. Bertani ${ }^{4}$, I. Tinazzi ${ }^{5}$, F. Martinis ${ }^{1}$, N. Girolimetto ${ }^{1}$, C. Salvarani ${ }^{1,3}$, M. Beltrami ${ }^{2}$, P. Macchioni ${ }^{1}$. ${ }^{1}$ AUSL-IRCCS Reggio Emilia, Rheumatology Department, Reggio Emilia, Italy; ${ }^{2}$ AUSL-IRCCS Reggio Emilia, Medicine department, IBD Unit, Reggio Emilia, Italy; ${ }^{3}$ Rheumatology Unit, University of Modena \& Reggio Emilia, Rheumatology Department, Modena, Italy; ${ }^{4}$ University of Modena \& Reggio Emilia, Gastroenterology Department, IBD Unit, Modena, Italy; ${ }^{5}$ Sacro Cuore Don Calabria Hospital, Negrar, Verona, Italy, Rheumatology Unit, Negrar, Italy

Background: Previous studies have reported an association between psoriasis (PsO) and inflammatory bowel disease (IBD). Coexistence of IBD and $\mathrm{PsO}$ has been associated with significant higher prevalence of enthesitis and dactylitis ${ }^{1}$.

Objectives: To compare the prevalence of clinical and ultrasonographic peripheral enthesis abnormalities in a consecutive series of patients with IBD and psoriasis ( $\mathrm{PsO}$ ) as compared to a group of IBD patients without psoriasis (IBD).

Methods: One-hundred seventy-four IBD consecutive patients [36 PsO and 138 IBD, M/F 91/83, mean age $42.6 \pm 14.7$ years, mean disease duration $110 \pm 12.3$ months] entered the study. A complete clinical examination, including rheumatological history, 66/68 peripheral joint count, MASES and LEI scores, BASDAI, and dactylitis count, was performed at study entry. Laboratory test (ESR, CRP, $\mathrm{Hb}$, fecal calprotectin) were collected. Axial or peripheral SpA diagnosis was made using ASAS criteria ${ }^{2}$

US examination was executed by a rheumatologist blind to clinical data, using an Esaote MyLabClass, $18-6 \mathrm{MHz}$ linear multifrequence transducer both in B-mode and PD-mode. The following sites were examined bilaterally: lateral epicondyle of the humerus, distal quadriceps femoris insertion into the patella, inferior pole of the patella, tibial distal insertion of the patellar tendon, calcaneal insertion of the Achilles tendon, and plantar aponeurosis insertion. Knee and ankle joints were evaluated for synovial hypertrophy, PD signal and fluid effusion. Enthesitis was defined according to OMERACT ${ }^{3}$ and scored as $0-36$ for GUESS and 0-136 for MASEI.

Results: PsO patients had later IBD onset (mean age $39 \pm 14.7$ vs $33 \pm 13.2$ in IBD group, $\mathrm{p}=0.02$ ). There weren't observed any significant differences in IBD duration and Crohn/UC prevalence.

No significant difference between the two groups in rheumatological history and clinical examination was detected, except for familiar history of psoriasis (PsO $44 \%$ vs IBD $16 \%, p<0.001)$. Prevalence of $\mathrm{SpA}$ was $33,3 \%$ in $\mathrm{PsO}$ group and $37 \%$ in IBD group $(\mathrm{p}=0.687)$.

146 patients $(83.4 \%)$ showed structural damage at $\geq 1$ enthesis, 44 patients $(25 \%)$ had at least 1 active enthesitis, with no significant difference between the two groups. PsO group showcased a significantly increased prevalence of patients having $\geq 1$ thickened enthesis $(86.1 \%$ vs $63.9 \%, p=0.009)$ and of PD signal at knee examination ( $11.1 \%$ vs $2.2 \%, p=0.034)$. Higher values of GUESS score were observed in PsO $(8.1 \pm 5.1$ vs $5.8 \pm 3.9, p=0.017)$ Enthesis hypoechogenicity was more prevalent in IBD group (27.5\% vs $11.1 \%$, $\mathrm{p}=0.049$ ) 